\title{
Imaging joints for calcium pyrophosphate crystal deposition: a knock to the knees
}

\author{
Robert Terkeltaub* \\ See related research by Abhishek et al., http://arthritis-research.com/content/14/5/R205
}

\begin{abstract}
With advanced age, articular calcium pyrophosphate crystal deposition (CPPD) is common. Defining who has CPPD is of growing importance, given increases in longevity in many countries and the frequent association of chondrocalcinosis with osteoarthritis. Chondrocalcinosis detected by plain radiography serves as a major screening tool, but how many and which sites to screen have not been adequately defined in the past. The work of Abhishek and colleagues in the previous issue of Arthritis Research and Therapy sheds new light on the incomplete information from knee radiographs, and helps position us to learn more about the epidemiology, pathophysiology, diagnosis, and clinical impact of CPPD.
\end{abstract}

With advanced age, calcium pyrophosphate crystal deposition (CPPD) is a common finding in many fibrocartilages and hyaline articular cartilages of larger joints, and in certain tendons and soft tissues [1-4]. There is continuing evolution in several of the factors associated with the development of CPPD in many countries, owing to increasing longevity of the population, increases in the prevalence of osteoarthritis (OA), of traumatic joint injury and of end-stage kidney disease, and partly due to changes in prescription patterns of medications that promote hypomagnesemia (for example, several diuretics, proton pump inhibitors, tacrolimus, cyclosporine) [3-5]. However, the prevalence of CPPD appears to vary in different regions - with, surprisingly, relatively infrequent knee and wrist CPPD in Beijing, China, despite elevated

\footnotetext{
*Correspondence: rterkeltaub@ucsd.edu

Rheumatology Section, San Diego Veterans Affairs Medical Center, Division of Rheumatology, Allergy and Immunology, University of California San Diego School of Medicine, VA Medical Center, 3350 La Jolla Village Drive, San Diego, CA 92161, USA
}

prevalence of knee OA in the Chinese in Beijing relative to whites in Framingham, MA, USA [6].

Identifying CPPD is gaining in importance. Methodology advances, such as the utility of high-resolution ultrasound, have attracted recent attention, yet chondrocalcinosis $(\mathrm{CC})$ detected by plain radiography remains the primary screening approach, with advantages including universal availability and panoramic imaging of the joint [2]. The notion, and hints from past studies, of a very high yield of screening for $\mathrm{CC}$ in the knees alone by plain radiography was particularly attractive [1]. To advance understanding of the epidemiology, pathophysiology, and clinical impact of CPPD, it is vital to define how many and which sites to screen by plain radiography. The work of Abhishek and colleagues in the previous issue of Arthritis Research and Therapy sheds new light on a previously murky area, by demonstrating, in an amply sized study, the incompleteness of CPPD information from knee radiographs alone (that is, a knock to the knees) [1].

Abhishek and colleagues performed a cross-sectional study of 3,170 subjects embedded in the Genetics of Osteoarthritis and Lifestyle (GOAL) study [1]. Each subject underwent knee, hand, and pelvis radiographs, and urine and blood sampling. In the GOAL study, approximately one-third of subjects had clinically severe hip OA, or clinically severe knee OA, or did not have knee or hip OA. Since this population is skewed for high prevalence of hip or knee OA, the distribution of CC was analyzed for the subgroup without radiographic hip or knee OA. Some findings in this subgroup of 1,000 subjects largely confirmed certain observations from smaller cohorts [1]. Specifically, knee CC most commonly involved the lateral compartment and the menisci, and the knee was the most common single site for radiographic CC. Knee CC in the GOAL study was followed in descending order of frequency by CC of wrists, hips, and then symphysis pubis. Interestingly, symphysis pubis calcification was 10-fold more common than hip CC in a previous, large, community-based study [7]. The relatively high prevalence of severe symptomatic hip or knee $\mathrm{OA}$ and the relatively young age of GOAL participants 
may have accounted for the relatively high prevalence of $\mathrm{CC}$ at the hips compared with the symphysis pubis seen in the GOAL study [1].

Other findings of the GOAL study included wrist CC being more common than hip CC, and, with the notable exception of hips, CC was most often bilateral at the individual joint loci studied [1]. Subjects with meniscal or hyaline articular cartilage $\mathrm{CC}$ of the knee were also of comparable age and did not demonstrate preferential association with fibrocartilage $\mathrm{CC}$ at distant joints. A skeletal system-wide disorder of inorganic pyrophosphate metabolism affecting multiple skeletal tissue types, associated with aging, metabolic, or uncommon genetic disease, appears central to the pathogenesis of CPPD $[3,8]$. Furthermore, dysregulated chondrocyte growth factor responsiveness and differentiation related to aging and/or OA are also probably involved [8]. The findings of the GOAL study [1] remind us of unsolved issues about articular region-to-region differences in phenotype of what still appears to be a skeletal system-wide disorder of aging in the majority of patients with CPPD.

In the GOAL study, $42 \%$ of CC cases had no knee involvement; that is, hip, wrist, and symphysis pubis CC, and calcification in tissues of the metacarpophalangeal joint consistent with CPPD, occurred quite commonly without radiographic knee CC. This result is quite different from most, but not all, prior reports [1]. The authors astutely suggest that this distinction may reflect overrepresentation of symptomatic knee arthropathy in previous, small, hospital-based studies. Their results and discussion also cogently point out that many subjects with CPPD would be erroneously classified if plain radiography screening was limited to either the knees or two of the three screening regions employed in the GOAL study (knees, hands, and pelvis).

The extent of what the results from Abhishek and colleagues' study mean for diagnosis is not yet clear. Certainly, the prevalence of $\mathrm{CC}$ at the knees, the knees and pelvis, and the knees and wrists reported in the GOAL study were similar to respective prevalence numbers reported in past community studies [1]. Nevertheless, the GOAL study has limits in general applicability, owing to the nested analysis involving re-attribution of case and control status within a much broader case-control cohort study designed to examine risk factors for large joint OA. Moreover, CC is not the sole radiographic feature of CPPD [9], and European League Against Rheumatism diagnostic recommendations include that 'radiographic CC supports the diagnosis of CPPD, but its absence does not exclude it' [2]. The European League Against Rheumatism also has pointed out in their CPPD recommendations that 'the presence of radiographic CC and advanced age increases this likelihood, but definitive diagnosis needs to be crystal proven'
[2]. CPPD can co-exist with other arthropathies that may be the primary symptomatic problem in a given patient, including not simply degenerative arthritis due to primary OA but also gout or pyogenic infection of the diseased CPPD joint. One clinically informative lesson of the GOAL study is that over $90 \%$ of cases with metacarpophalangeal joint calcification in the GOAL study had CC in other joints [1]. In the absence of CC, soft tissue calcification, or other radiographic changes suggestive of CPPD in an affected joint, how productive or cost-effective it is to survey remote joints by radiography to look for the relatively common abnormality of $\mathrm{CC}$ remains unclear.

In conclusion, the GOAL study is the largest yet to examine how $\mathrm{CC}$ and metacarpophalangeal joint calcification are distributed [1]. In particular, the work highlights the incompleteness of information from knee radiographs. Further ultrasound $[2,10]$ and macro-radiographic studies of CPPD, and examination of the urine and blood samples drawn in the GOAL study, as well as large community-based cross-sectional studies, should be informative for analyses of both CPPD and OA. There remains much to learn about the epidemiology and clinical impact of CPPD by itself and with primary OA.

\section{Abbreviations}

CC, chondrocalcinosis; CPPD, calcium pyrophosphate crystal deposition; GOAL, Genetics of Osteoarthritis and Lifestyle; OA, osteoarthritis.

\section{Acknowledgements}

Supported by the VA Research Service and the National Institutes of Health (PAG07996).

\section{Competing interests}

The author declares that he has no competing interests.

Published: 27 December 2012

\section{References}

1. Abhishek A, Doherty S, Maciewicz R, Muir K, Zhang W, Doherty M: Chondrocalcinosis is common in the absence of knee involvement. Arthritis Res Ther 2012, 14:R205.

2. Zhang W, Doherty M, Bardin T, Barskova V, Guerne PA, Jansen TL, Leeb BF, Perez-Ruiz F, Pimentao J, Punzi L, Richette P, Sivera F, Uhlig T, Watt I, Pascual E: European League Against Rheumatism recommendations for calcium pyrophosphate deposition. Part l: terminology and diagnosis. Ann Rheum Dis 2011, 70:563-570.

3. Abhishek A, Doherty M: Pathophysiology of articular chondrocalcinosis role of ANKH. Nat Rev Rheumatol 2011, 7:96-104.

4. Guerne PA, Terkeltaub R: Calcium pyrophosphate dihydrate crystal deposition: epidemiology, clinical features, diagnosis, and treatment. In Gout and Other Crystal Deposition Arthropathies. Edited by Terkeltaub R. Philadelphia, PA: Elsevier; 2011:249-265.

5. Rho YH, Zhu Y, Zhang Y, Reginato AM, Choi HK: Risk factors for pseudogout in the general population. Rheumatology (Oxford) 2012, 51:2070-2074.

6. Zhang Y, Terkeltaub R, Nevitt M, Xu L, Neogi T, Aliabadi P, Niu J, Felson DT: Lower prevalence of chondrocalcinosis in Chinese subjects in Beijing than in white subjects in the United States: the Beijing Osteoarthritis Study. Arthritis Rheum 2006, 54:3508-3512.

7. Ramonda R ME, Perissinotto E, Sartori L, Punzi L, Corti MC, Hirsch R, Manzato E, Zambon S, Baggio G, Crepaldi G: Prevalence of chondrocalcinosis in Italian subjects from northeastern Italy. The Pro.V.A.(PROgetto Veneto Anziani) study. Clin Exp Rheumatol 2009, 27:981-984. 
8. Terkeltaub R, Pritzker KPH: Pathogenesis and molecular genetics of calcium pyrophosphate dihydrate crystal deposition disease. In Gout and Other Crystal Deposition Arthropathies. Edited by Terkeltaub R. Philadelphia, PA: Elsevier; 2011:240-248.

9. Resnick D, Niwayama G, Goergen TG, Utsinger PD, Shapiro RF, Haselwood DH, Wiesner KB: Clinical, radiographic and pathologic abnormalities in calcium pyrophosphate dihydrate deposition disease (CPPD): pseudogout. Radiology 1977, 122:1-15.

10. Frediani B, Filippou G, Falsetti P, Lorenzini S, Baldi F, Acciai C, Siagkri C, Marotto
D, Galeazzi M, Marcolongo R: Diagnosis of calcium pyrophosphate dihydrate crystal deposition disease: ultrasonographic criteria proposed. Ann Rheum Dis 2005, 64:638-640.

doi:10.1186/ar4097

Cite this article as: Terkeltaub R: Imaging joints for calcium pyrophosphate crystal deposition: a knock to the knees. Arthritis Research \& Therapy 2012

14:128. 